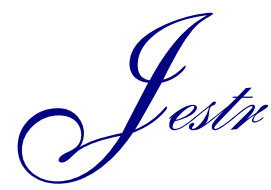

\title{
Perpetual Motion Machine
}

\author{
D. Tsaousis* \\ School Adviser of Natural Science Teachers of Ioannina8 Seferi street, Eleoussa, 455 00,Ioannina Hellas . \\ Received 30 April 2008; Accepted 17 September 2008
}

\begin{abstract}
Ever since the first century A.D. there have been relative descriptions of known devices as well as manufactures for the creation of perpetual motion machines. Although physics has led, with two thermodynamic laws, to the opinion that a perpetual motion machine is impossible to be manufactured, inventors of every age and educational level appear to claim that they have invented something «entirely new» or they have improved somebody else's invention, which «will function henceforth perpetually»! However the fact of the failure in manufacturing a perpetual motion machine till now, it does not mean that countless historical elements for these fictional machines become indifferent. The discussion on every version of a perpetual motion machine on the one hand gives the chance to comprehend the inventor's of each period level of knowledge and his way of thinking, and on the other hand, to locate the points where this "perpetual motion machine» clashes with the laws of nature and that's why it is impossible to have been manufactured or have functioned. The presentation of a new «perpetual motion machine» has excited our interest to locate its weak points. According to the designer of it the machine functions with the work produced by the buoyant force
\end{abstract}

Keywords: Perpetual motion machine, heat engine, efficiency.

\section{Introduction}

Perpetual motion machine: A machine which, since set in function, continues to function perpetually without supplying any energy.

The question about the perpetual motion machine is one of the issues, which attracts people who tend to believe strange things and occultism. That's why such ideas are adopted from various non-recognized religious circles which often describe in their books or in their speeches perpetual motion machines, which however have neither been manufactured nor have functioned. Usually the members of these organizations ignore the fundamental laws of physics and surely they are not the researchers who possess the knowledge to improve or generalize the laws. On the other side it must be stated that it is not always easy to be proved theoretically that it is impossible for a manufacture to function because in each more complicated system a great number of secondary or inconspicuous activities are involved, which in energy issues should be taken into consideration.

There is also a great number of ideas about perpetual motion machines, which (don't) function with magnets, chemical substances or flame. The «eternal light» without the addition of fuel seems to have been cultivated through the centuries mainly in religious circles. Since the first century A.D. there have been relative descriptions of the Roman military officer, politician, natural philosopher and historian Gaius Plinius Secundus (23 - 79 A.D.). In the

\footnotetext{
*E-mail address: dtsaousis@sch.gr

ISSN: 1791-2377 @ 2008 Kavala Institute of Technology. All rights reserved.
}

website of Hans-Peter Gramatke there is a detailed presentation of the most known devices for the designing of a perpetual motion machine with pictures, assimilated movements e.t.c.

As an example, here is a plumbing - mechanic system with communicating tubes of different length as Fig.1, which contains two liquids with an important difference in density e.g. water and mercury. The globules that will be moving perpetually in the two tubes, float in both liquids. Each globule that goes upwards because of the buoyant force in the left tube falls onto the wheel, which rotates because of each globule's momentum, while afterwards drops into the right tube. There the increased total weight of the globules pushes the formed column and in this way another globule reaches the bottom and then it rises to the surface of the liquid in the left tube, and so on. Even if this device is not possible to function as a perpetual motion machine, the factual cause seems not easy to be located.

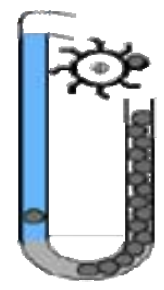

Fig..1. Perpetual motion machine consisting of a plumbing - mechanic system 


\section{Historical elements}

The idea of the perpetual motion machine appears for the first time in the East and to be exact in the $12^{\text {th }}$ century A.D. in India. In ancient Greece and in Rome, but also during the later Antiquity it hasn't been reported a perpetual motion machine not even as a theoretical version.

The Indian Mathematician and Astronomer Bhaskaracharya $(1114$ - 1185) describes a perpetual motion mechanism as Fig.2, in one of his literary works with the following words: "The machine rotates at full speed because the mercury is at the one side of the wheel nearer the axis and farther from the other side». The apparatus which Bhaskaracharya describes was manufactured by a lot of subsequent researchers in the same form or in different versions and of course it didn't constitute a perpetual motion machine. The simplest of these manufactures consists of a wheel in the perimeter of which less or more complicated arms are attached and which change the center of mass, during the rotation. While an impression of a perpetual motion machine may be given visually, in fact the system balances at some moment. On the other hand nowadays, we know that the rotating wheel heats the axis due to friction and it must also overcome the drag with the result after some rotations the energy caused by the initial external propulsion will be consumed and so the rotation stops.

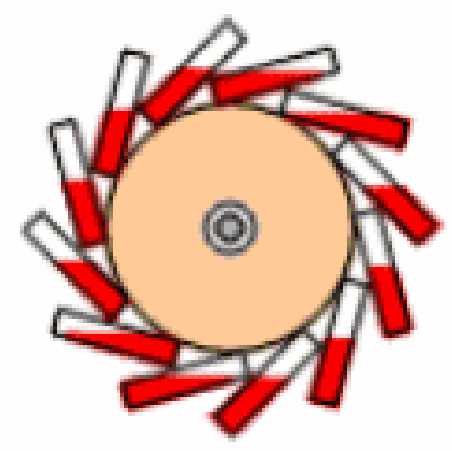

Fig. 2. Bhaskaracharya's perpetual motion mechanism

A notebook of Villard de Honecourt aged back to the $13^{\text {th }}$ century has been rescued. In this notebook he presents several magnificent buildings and a series of machines, among them a perpetual motion machine with masses (hammers), which change the center of mass during its rotation. It is not known whether these designs of Honecourt were ever accomplished or not, but for sure the perpetual motion machine didn't work because it is a version of Bhaskaracharya's conception. During the Renaissance De Georgio, Leonardo da Vinci and Vittorio Zonca designed or tried to manufacture a perpetual motion machine. Of the three above-mentioned Leonardo is of the opinion that the function of a perpetual motion machine belongs to the field of the impossible and he identifies the researchers of perpetual motion machine with the Alchemists: «You researchers of the perpetual motion, how many conceited, fictional works haven't you created carrying out your researches.... You had better make company with the creators of gold».

In the centuries that followed more and more names of researchers for the perpetual motion machine were added till Johann Bessler, who around 1715 presented to his astonished audience a perpetual motion machine, which was functioning for a several period of time, but information states that it proved to be a great deceit and Bessler disappeared. There aren't any other details known about this subject.

In the bibliography of Middle Ages and Renaissance the perpetual motion machines are referred as: perpetuum mobile naturae and perpetuum mobile physicae. The first category concerned systems of the nature (sun, stars, seasons of the year and so on), which were considered as perpetually moving mainly because they functioned with God's will. In the second category belonged the systems which man would make following as example the divine creations. These aspirations of the researchers were considered then, sometimes, to be a recognition of the divine deed and an effort of its imitation and sometimes they are insulting. Those occupied with such subjects would be presented before the «Inquisition» and be sentenced to death.

Anyway, in 1775 the French Academy of Sciences decided not to accept suggestions about perpetual motion machines any more.

\section{Definitions}

First kind of perpetual motion machine: Every machine which functions perpetually and produces work, without an input of external energy in any form and without being subjected to any decay as time passes as far as its components and materials are concerned.

Second kind of perpetual motion machine: Every machine in periodical function, which converts totally heat energy into other type (mechanic, electric e.t.c.).

Third kind of perpetual motion machine: The same as the first kind, without producing work.

Perpetual motion machine of first kind would be a pair of motor - generator where the generator supplies electric energy in return for its motion without any loss.

Perpetual motion machine of second kind could be every system, which would use for its function the heat of the environment e.g. a vehicle which would move exploiting the heat of the air.

Finally, perpetual motion machine of third kind can be considered a system sun - planets or every nucleus of an atom with its electrons, which seem to function without exchange of energy with their environment, thing which is not right.

\section{Thermodynamic Laws}

With the statement of the thermodynamic laws in the middle of the $19^{\text {th }}$ century the creation of a perpetual motion machine was theoretically excluded.

The equation

$$
Q=\Delta U+W
$$

Consists the first law of thermodynamics

The quantity of energy supplied to any isolated system in the form of heat $Q$ is equal to the work $W$ done by the system plus the change in internal energy $\Delta U$ of the system. 
The first law of thermodynamics is the application of the principle of the conservation of energy, which is valid for every isolated system.

The thermal efficiency, e, of the heat engine is defined as

$e=\frac{\text { work done during one cycle }}{\text { heat added during one cycle }}=\frac{W}{Q_{h}}$

The net amount of heat $\mathrm{Q}$, which is absorbed by the substance, is the amount of heat it receives from the high temperature heat source $\mathrm{Q}_{\mathrm{h}}$ minus that one which it exhausts to the low temperature heat sink $\mathrm{Q}_{\mathrm{c}}$. The work produced by the gas equals with the net amount of heat it absorbs that is

$\mathrm{W}=\mathrm{Q}_{\mathrm{h}}-\left|\mathrm{Q}_{\mathrm{c}}\right|$

Replacing Eq 3 in the Eq 2 we have:

$$
e=\frac{Q_{h}-\left|Q_{c}\right|}{Q_{h}} \quad \text { or } \quad e=1-\frac{\left|Q_{c}\right|}{Q_{h}}
$$

It is interesting to note that the efficiency of steam engines has increased from $0.17 \%$ for the first steam engines of the seventeenth century to over $40 \%$ for the turbines used in modern power plants.

From the Eq 4 we see that the thermal efficiency of an operating heat engine must always be less than $100 \%$. It would be $100 \%$ if the engine transformed the whole amount of heat to work. So far nobody managed to manufacture such an engine. All engines exploit only a part of the heat and they exhaust a notable amount of heat to the environment. The repeated failures of the researchers to manufacture a «perfect» heat engine which would transform completely the heat to available work convinced us that this incapability is due to restriction set by the nature itself. This finding out led to the formulation of the second thermodynamic law by Kelvin and Plank:

It is impossible to extract an amount of heat from a hot reservoir and use it all to do work. Some amount of heat must be exhausted to a cold reservoir.

Speaking about heat, it flows spontaneously from a high temperature object to a lower temperature object. The reverse course demands consumption of energy. A heat pump is a device which applies external work to extract an amount of heat from a cold reservoir and delivers heat to a hot reservoir. A refrigerator is a heat engine in which work is done on a refrigerant substance in order to collect energy from a cold region and exhaust it to a higher temperature region, thereby further cooling the cold region. The statements about refrigerators apply to air conditioners and heat pumps, which embody the same principles. However for the function of these machines we spend energy. It is impossible to manufacture a refrigerator, which can function without consuming energy. This finding out led to the "second form" or Clausius statement of the second law.

It is not possible for heat to flow from a colder body to a warmer body without any work having been done to accomplish this flow.

The two forms of the second thermodynamic law, which apparently are entirely unlinked, are equal in value. If one of them is true the other one will be true, too.

The first law is the application of conservation of energy to the system, and the second sets limits on the possible efficiency of the machine and determines the direction of energy flow. According to the second law though, nature sets restrictions in the transformation of one kind of energy to another one. Heat cannot be transformed $100 \%$ to mechanic energy. Also the second thermodynamic law defining that the heat is always transmitted from the warmer to the colder body, defines the direction towards which the phenomena happen spontaneously in nature.

In 1824 the French engineer Sadi Carnot described a reversible cyclic process which, was called Carnot Cycle. The Carnot cycle can be thought of as the most efficient heat engine cycle allowed by physical laws. The Carnot efficiency sets the limiting value on the fraction of the heat, which can be so used. Such a supposed idealized machine is called Carnot engine and its output constitutes the superior limit for the output of all the other machines. This deduction is known as Carnot theorem:

The efficiency of a Carnot engine or Carnot efficiency is the maximum efficiency possible for a heat engine working between two given temperatures.

It is proved that the Carnot efficiency is:

$e_{\text {Carnot }}=\frac{T_{h}-T_{c}}{T_{h}}=1-\frac{T_{c}}{T_{h}}$

The ratio between the work done and the amount of heat introduced into a system going through a Carnot cycle, the Carnot efficiency, is equal to the difference between the two temperatures of the isothermal steps of the cycle divided by the higher of the two temperatures.

The result states that the Carnot efficiency depends only on the temperatures of the two heat tanks. It is big when the temperature difference is great and it is very small when the temperatures differ a little. Since most of the practical applications have as cold tank the environment, that is the temperature of about $300^{\circ} \mathrm{K}$, the higher is the temperature of the body which «emits» heat, the more profitable may be its exploitation. Also the result confirms the second thermodynamic law. In order to have Carnot efficiency $100 \%$ we must have $T_{c}=0$, which is impossible.

\section{Criterion of success for a perpetual motion machine}

Although physics has led with the two thermodynamic laws to the opinion that it is impossible to manufacture a perpetual motion machine, researchers of every age and educational level appear, claiming that they have found something «entirely new» or that they have improved the invention of somebody else's, which «will function for ever henceforth» Executives in research centers and educational institutions very often face persistent visitors with ideas of perpetual motion machines.

Firstly the rules of physics, which we call axioms, are simply principles that are deductions, which are confirmed in every measurement and every calculation. Because, therefore the universality and the general acceptance of these principles, we consider that they hold a place of axiom that is they constitute fundamental affairs, which don't need to be proved. Contrary to the «unsolved» problems of Euclid's Geometry (trisection of the acute angle, squaring the cycle e.t.c.) which evidently are not solved with the predetermined rules, the axioms of physics are empirical principles, which 
perhaps some time will be proved to be of limited validity in space or in time.

On the one side, therefore, we have with absolute certainty the repetition of the same results in an enormous number of measurements and calculations. And on the other side in science there is the possibility of subversion or as it is usually happens the extensions and generalization of some deductions, which are considered obvious today. Of course, criterion of success for a machine is not whether it obeys the $1^{\text {st }}$ or the $2^{\text {nd }}$ law of physics or not, but whether it functions. That is if it does what its manufacturer claims. With this criterion we are in position to declare in advance that till today a perpetual motion machine hasn't been materialized despite the countless efforts, theoretical and constructional ones.

Thus, every claim that a «new scientific theory proves» the possibility of function of perpetual motion machines e.g. with the introduction of new concepts, which are unknown in physics, such as the discussed free energy and so on, is false-scientific. From the other side, the fact that up to now (and with all certainty in the predictable future) failure in manufacturing a perpetual motion machine doesn't mean that the countless historical elements for these fictional machines are vain. The discussion on any version of perpetual motion machine gives the opportunity on the one hand to comprehend the level of knowledge and the way of thinking of the researchers of each period and on the other hand, to locate the points in which this «perpetually moving machine» clashes with the natural laws and that's why it has been impossible to be manufactured or be in function.

\section{A perpetual motion machine which «functions» with buoyant force}

There have been a lot of efforts to manufacture a perpetual motion machine concerning the production of work with the use of the buoyant force. Motive for the following analysis was a new effort of manufacturing a perpetual motion machine, which we prove theoretically, that can't function.

\subsection{Theoretical introduction}

Supposing we have a container filled with a liquid of density $\mathrm{d}$, the free surface of which is at a height $\mathrm{h}$ from the bottom. On the bottom of the container there is a parallelepiped of negligible length as in fig. 3 . We are going to calculate the work produced during the shift to length $\mathrm{L}$, of a side with area $\mathrm{S}$ of the elementary parallelepiped of null initial volume, which is in the container at a depth $\mathrm{h}$, until the parallelepiped acquires volume $\mathrm{V}$.

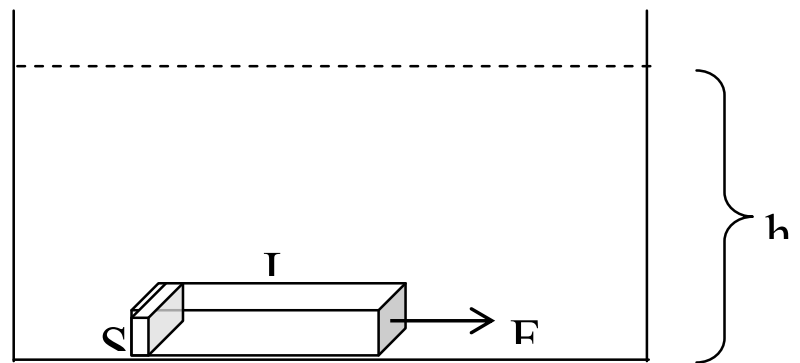

Fig. 3. Work produced by F, overcoming the force caused by pressure
The force $\mathrm{F}$ that we will apply should be so much that would be able to overcome the force caused by the pressure at the depth $\mathrm{h}$, that is

$$
\mathrm{F}=\mathrm{PS}
$$

And the consumed work will be:

$$
\mathrm{W}_{1}=\mathrm{FL} \text { or } \mathrm{W}_{1}=\mathrm{PSL} \text { at last } \mathrm{W}_{1}=\mathrm{dghV}
$$

If we let the parallelepiped to rise to the surface of the liquid a work will be produced due to the buoyant force:

$$
\mathrm{W}_{2}=\mathrm{Ah} \quad \text { or } \quad \mathrm{W}_{2}=\mathrm{dg} \mathrm{Vh}
$$

From the Eq 7 and 8 the result is that

$\mathrm{W}_{1}=\mathrm{W}_{2}$

\subsection{Description of the machine}

The perpetual motion machine that we are examining is composed of a circular disc to which we have adapted weightless n parallelepipeds, as fig. 4. During the rotation of the disc the parallelepipeds can pass from the lower part of a container which is filled with a liquid via a suitable mechanism so that the liquid cannot slop away. According to the designer the buoyant force is exerted on the parallelepipeds when they are in the liquid and so the buoyant force will be the moving force for the production of energy.

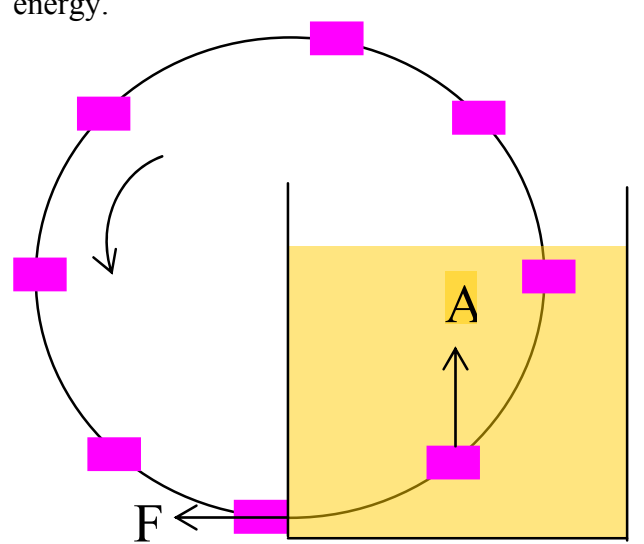

Fig. 4. A circular disc, to which we have adapted $n$ weightless parallelepipeds, is rotating while a part of it is submerged in a container filled with a liquid.

In fact for each rotation of the disk the buoyant force A produces a work equal with $\mathrm{nW} 1$. For each rotation, however, is consumed also work for the submersion of the parallelepipeds in the container equal with $\mathrm{nW}_{2}$ in order to overcome force the $\mathrm{F}$ because hydrostatic pressure.

But because of Eq 9 we have

$\mathrm{nW}_{1}=\mathrm{nW}_{2}$

Therefore the kinetic energy of the disc is not altered, since as much work is produced so much is consumed. That is to say that the machine does not produce any energy.

The error of the designer of the particular machine is that he did not take into consideration the force $\mathrm{F}$ because of the hydrostatic pressure and the work that will be consumed for the submersion of the parallelepipeds in the container. 
In the whole analysis we made we did not include, by no means, frictions. However frictions exist and they will consume any initial kinetic energy we give to the disc with result after a little time it stops.

\section{Our perpetual motion machine}

The study we have done for the perpetual motion machines gave us the opportunity to see some designs and conceptions of them. Some of these machines, although don't function, impressed us for the imagination of their designers. We were also occupied with the educational use of such a machine. We thought then to materialize some design of such a machine, not with purpose to research whether this machine works or not, but to help the students, who based on its not functioning, consolidate the conservation of energy and validity of the two thermodynamic laws.

So we manufactured a perpetual motion machine based on the design of fig.5, which describes the Arabian perpetual motion machine (Arabian Perpetuum Mobile) which is a version of Bhaskaracharya's machine. Our manufacture is shown in the fig. 6 .

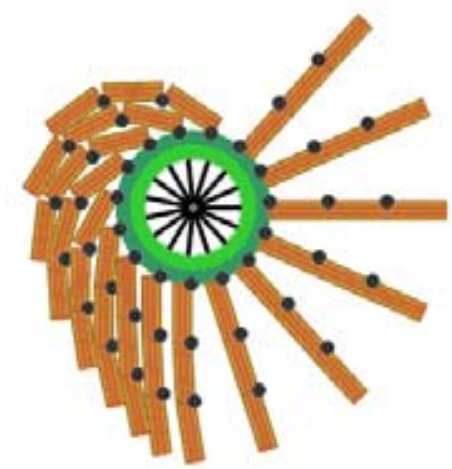

Fig. 5. Arabian Perpetuum Mobile.

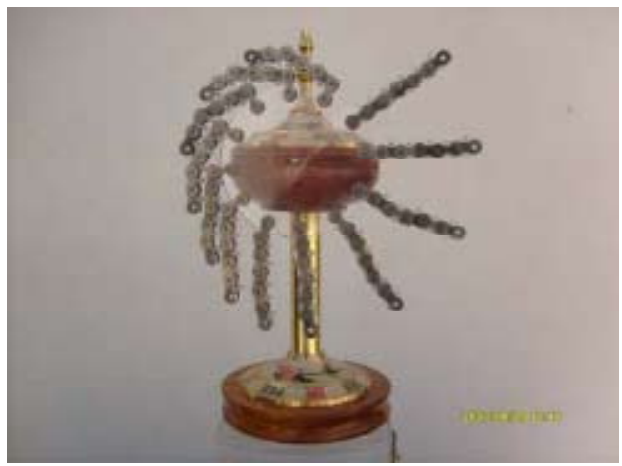

Fig. 6. Picture of our perpetual motion machine, which is a version of Arabian Perpetuum Mobile.
The stems of the Arabian Perpetuum Mobile which fold only towards the one direction were replaced by parts of a bicycle chain, which were adapted on a disc made of Plexiglas. At the nodes we have tied up nylon joints so that the chain can fold only towards one side as it shown in fig 7 .

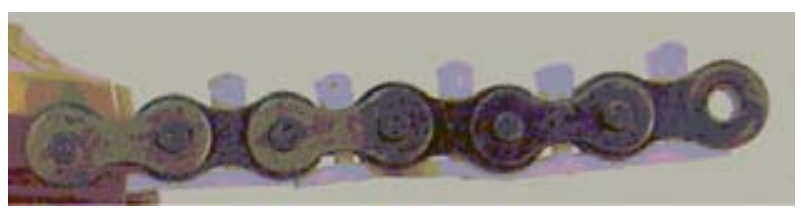

Fig. 7. Details concerning fig. 6 .

The result was astonishing. The students are influenced and express the view that the machine will rotate, although they have been taught the conservation of energy. After discussion they understand their error, and so they consolidate their knowledge on the law of conservation of energy.

Note: The present version is an inept translation of the original Greek manuscript.

Acknowledgments: We wish to thank our collaborator in this work, Georgiou Athanasios, School Adviser of English Teachers, for his help in translating the initial Greek essay into English.

\section{References}

1. Marketou Pilarinou Maria, Lessons of General Physics, Issue I, Thermodynamics, Thessaloniki (1967).

2. Ikomomou N., Introduction in Physics, Issue II, Oıкоvó Thessaloniki (1968).

3. Aleksopoulou K. D. \& Marinou D. I. Physics, Issue I, Athens (1980).

\footnotetext{
4. Ioannou A., Ntanos I.,Pittas A., Raptis S, Physics, Form B of the Senior High School, Athens, (2000).

5. http://sfrang.com/historia/parart089.htm

6. http://www.richardclegg.org/htdocs/perpetual/torus.html
} 